\title{
Optoelectronic Properties of GaN-Based Light-Emitting Diodes with Different Mesa Structures
}

\author{
Xueyun Song ${ }^{1,2}$, Xianghua Zeng ${ }^{1 *}$, Junbin Zhang ${ }^{1}$, Yuzhe Jin ${ }^{1}$, Xiangdong Meng ${ }^{1}$ \\ ${ }^{1}$ College of Physics \& Technology, Yangzhou University, Yangzhou, China; ${ }^{2}$ Jiangsu Research Center of Engineering and Technol- \\ ogy for New Photosorce Solid-State Lighting, Yangzhou, China. \\ Email: *xhzeng@yzu.edu.cn
}

Received September $2^{\text {nd }}, 2012$; revised October $3^{\text {rd }}, 2012$; accepted November $4^{\text {th }}, 2012$

\begin{abstract}
GaN/InGaN lighting-emitting diodes with different mesa structures are studied. The current-voltage characteristics, light output power, luminous efficiency, and peak wavelength of the $\mathrm{GaN} / \mathrm{InGaN}$ lighting-emitting diodes with different mesa patterns are compared. It shows that the current-voltage characteristics of the chips with more mesa areas are improved greatly by reducing the current crowding. With higher injection current the light output powers of GaN-based LED with more mesa areas are enhanced. And the chips with more P-electrode area have a smaller red shift and a little bit blue shift due to the reduction of the current crowding.
\end{abstract}

Keywords: GaN-Based Leds; Mesa Structure; Optoelectronic Properties

\section{Introduction}

With small volume, light weight and long lifetime advantages, GaN-based LED has a wide range of applications, such as automobiles, backlighting, traffic lights, etc. It can be fabricated to white LED by many approaches, and expected to become the new fourth-generation solidstate lighting [1-5]. But most GaN-based LEDs have an insulating sapphire substrate, so their $\mathrm{P}$ and $\mathrm{N}$ electrodes are on one side, which inevitably produce current crowding effect [6-9] and result in an uneven distribution of current, serious heating effect, lower light extraction efficiency and output power, and severe red shifted peak of emission wavelength [10-13].

There are many methods to improve the output efficiency [14-17], but fabricating GaN-based LEDs with less current crowding has been an objective of researchers engaged in the filed $[6,13]$. And it is a good method to reduce the current crowding by optimizing the chip's electrode which will make the current distribute uniformly. In Ref [11], they reported on the efficiency of GaN-based LED with different mesa patterns, but they only designed the finger interdigitated patterns. This paper reports on the efficiency of GaN-Based LEDs with different mesa structures, here the mesa patterns have a better symmetry and some of them are more complicated (as shown in Figure 1), therefore more results are presented. By comparing the photoelectric parameters of

"Corresponding author. these samples, such as current-voltage (V-I) characteristics, output power, luminous efficiency, peak and dominant wavelength, we obtain that the mesa area or the Pelectrode area is directly related to the performance of the chips. On the one hand, the chips with more mesa area have a better V-I characteristics, on the other hand, as the mesa area will prevent the light emission, the lightemitting efficiency will decrease a little bit smaller, the output power and luminous efficiency are either. Therefore, it needs to compromise between optical and electrical properties in order to obtain more favorable optoelectronic performance of LEDs.

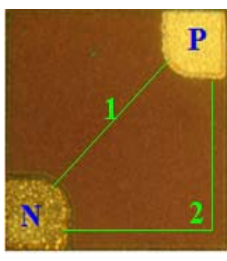

(a)

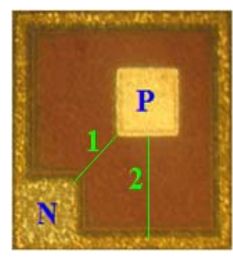

(d)

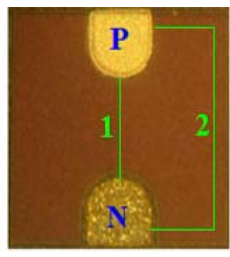

(b)

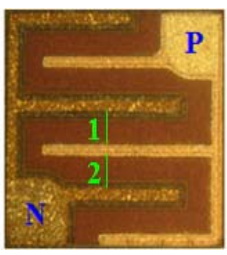

(e)

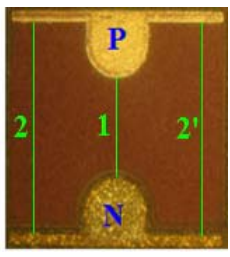

(c)

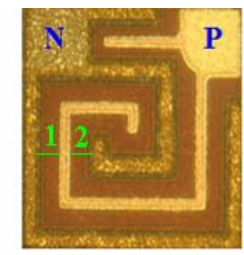

(f)
Figure 1. Micrograph of InGaN/GaN LEDs' with different electrodes. (a) \#1; (b) \#2; (c) \#3; (d) \#4; (e) \#5; (f) \#6. 


\section{Heterostructure and Chip Design}

GaN Based LEDs, which are grown by metal organic chemical vapor (MOCVD) on c-plane (0001)-oriented 2 inch sapphire substrate, consist of a thick undoped GaN buffer layer, an highly conductive $\mathrm{n}-\mathrm{GaN}$ lower cladding layer, an $\mathrm{InGaN} / \mathrm{GaN}$ multiple quantum well (MQW) active region, a p-GaN upper cladding and highly doped contact layer. Top-emitting LEDs with size of $356 \mu \mathrm{m} \times$ $356 \mu \mathrm{m}$ were fabricated using standard photolithography and $\mathrm{Cl}_{2} / \mathrm{SiCl}_{4} / \mathrm{Ar}$ inductively coupled plasma etching for current isolation purposes. Firstly, the p-GaN and active region were partially etching by an ICP etcher to expose an $\mathrm{n}-\mathrm{GaN}$ lower cladding layer for electrode formation. Subsequently, a $350 \mathrm{~nm}$ thick ITO was deposited on the $\mathrm{p}-\mathrm{GaN}$ top layer as transparent conductive layer (TCL). And $\mathrm{Cr}-\mathrm{Au}$ metals were deposited using the electronbeam evaporation system for the $\mathrm{P}$ and $\mathrm{N}$ electrodes. Finally, $\mathrm{SiO}_{2}$ was deposited as a passive layer to protect chips by plasma enhanced chemical vapor deposition (PECVD).

The masks make up of the basic unit, and each unit contains six different electrode shapes, each shape with 13 dies. The epitaxial wafer within basic unit size should have a good consistency, so comparing performance of chips with different electrodes are more reasonable within the basic unit. For simplicity, the LEDs corresponding to the six mesa patterns are called the samples \#1 - \#6 in sequence, their corresponding parameters, such as Mesa area, P-electrode area, Light-emitting area, and P-N electrodes separation for six samples are presented in Table 1, where the P-N electrodes separation is a typical length between $\mathrm{P}$ and $\mathrm{N}$ electrode as the labeled number one in Figure 1. The light output power of packaged LEDs was measured in an integrating sphere at constant temperature of $T=298 \mathrm{~K}$ in a dc current mode.

\section{Results}

Figure 2 shows the measured voltage-current (V-I) char-

Table 1. The parameters of the Mesa structures. The die size of all LEDs is $356 \mu \mathrm{m} \times 356 \mu \mathrm{m}$.

\begin{tabular}{|c|c|c|c|c|c|}
\hline \multirow{2}{*}{\multicolumn{2}{|c|}{ Samples Mesa area }} & \multirow{2}{*}{$\begin{array}{l}\text { P-electrode } \\
\text { area }\end{array}$} & \multicolumn{2}{|c|}{ Light-emitting area } & \multirow{2}{*}{$\begin{array}{c}\text { PN electrodes } \\
\text { spacing }\end{array}$} \\
\hline & & & \multicolumn{2}{|c|}{$\overline{\text { Mesa top Mesa sidewalls }}$} & \\
\hline & $\left(\mu \mathrm{m}^{2}\right)$ & $\left(\mu \mathrm{m}^{2}\right)$ & $\left(\mu \mathrm{m}^{2}\right)$ & $\left(\mu \mathrm{m}^{2}\right)$ & $(\mu \mathrm{m})$ \\
\hline$\# 1$ & 115410 & 9463 & 105948 & 1678 & 221 \\
\hline$\# 2$ & 115084 & 8925 & 106159 & 1911 & 156 \\
\hline$\# 3$ & 109184 & 12765 & 96419 & 1791 & 156 \\
\hline$\# 4$ & 86411 & 10000 & 76411 & 1469 & 107 \\
\hline$\# 5$ & 88233 & 23510 & 64723 & 2737 & 54 \\
\hline$\# 6$ & 77466 & 22168 & 55299 & 2653 & 43 \\
\hline
\end{tabular}

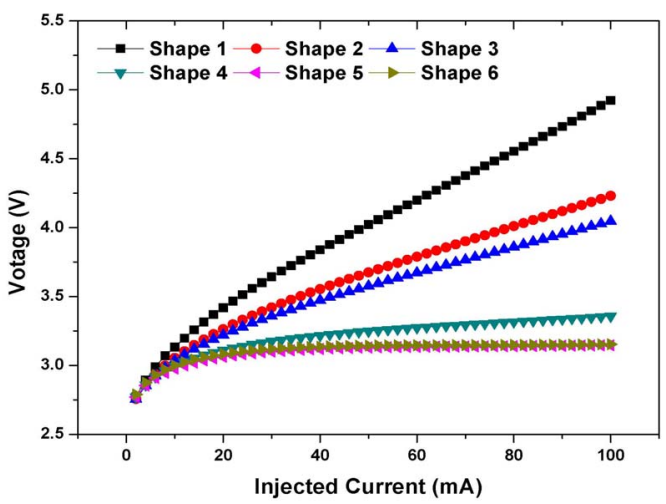

Figure 2. V-I characteristics of six samples.

acteristics of the LEDs. For six LEDs (samples \#1 - \#6), the forward voltages at $20 \mathrm{~mA}$ are $3.419,3.264,3.221$, $3.110,3.062,3.084 \mathrm{~V}$, respectively. With the mesa area increase, at $20 \mathrm{~mA}$ the voltages decrease, and the smallest one is $10 \%$ lower than the conventional mesa pattern. And the series resistances from the minim value of the derivative in V-I curves are 17.75, 11.12, 9.38, 2.12, 0.25 and $0.13 \Omega$, respectively, where the last one is only $0.70 \%$ of the first. From the separations between $\mathrm{P}$ and $\mathrm{N}$ electrodes in Table 1, it shows that the smaller the separation of PN electrodes, and the series resistance will decrease, so the LED V-I characteristics can be improved greatly. As a great current will crowd at the contact electrode edges, and results in a non-uniform temperature distribution over the die. This result is consistent with the calculations of Ref [13]. For the latter three mesa patterns, the P-contact areas are larger which lead to a small current density on the P-electrode areas, and the separations between $\mathrm{P}$ and $\mathrm{N}$ electrodes are smaller which lead to a more uniform current distribution. Comparing the last two mesa patterns, both the separations between $\mathrm{P}$ and $\mathrm{N}$ electrodes and the P-electrode areas are approximate, so their voltages are almost same. The reason is that the Indium-Tin Oxide (ITO) transparent conductive layer (TCL) which has much better conductivity will spread the current uniformly on its $p$-type surface, and the effects of the V-I characteristics on the separation and on P-electrode area are small. The voltage-current characteristics for latter three kinds of samples are much better with increasing injection current, and the voltages show saturation with injection current over $30 \mathrm{~mA}$.

Figure 3 shows the light output powers of the six samples as a function of injection current. The light output powers increase with increasing injection current, at 20 $\mathrm{mA}$ the light output powers for the six samples are 15.49, $15.08,14.67,13.49,11.55$ and $10.28 \mathrm{~mW}$, respectively, which decrease gradually with the mesa area. The chips corresponding to more P-electrode area have a smaller light output powers, as the more electrode area will countervail the efficient light-emitting area, so the light output 
powers decrease. At the same time the chips having more uniform current distribution will have a lower forward voltage, therefore input power is relatively lower. But for sample \#1 after $60 \mathrm{~mA}$, due to the current crowding effects the light output power decreases.

Figure 4 gives the changes of the measured luminous efficiency with injection current for six samples. With increasing injection current, the external quantum efficiency drops rapidly, at $20 \mathrm{~mA}$ their luminous efficiencies are $11.37,11.73,11.42,10.35,8.82$ and $7.641 \mathrm{~m} / \mathrm{mW}$, respectively. From samples \#1 to \#6, the luminous efficiency decreases gradually, as their top light emitting areas become gradually smaller. But the luminous efficiency of sample \#1 drops faster than others, because the uneven distributions of the current density induce the current crowding effect, and the thermal effect of the chips will reduce the luminous efficiency at the higher current $[12,13]$. Therefore, by optimizing mesa structure the luminous efficiency droop at high injection current can be abated.

The changes of the peak wavelength with injection current for six samples are shown in Figure 5. With increasing injection current, the wavelengths firstly drop down (blue shift), and up to $50 \mathrm{~mA}$ reach a minimum, and then increase gradually (red shift). The appearance of the blue or red shift for the peak wavelength may result from the existence of polarization filed in MQWs and heat effect of $\mathrm{GaN}$ material $[18,19]$. Over $50 \mathrm{~mA}$ a fast

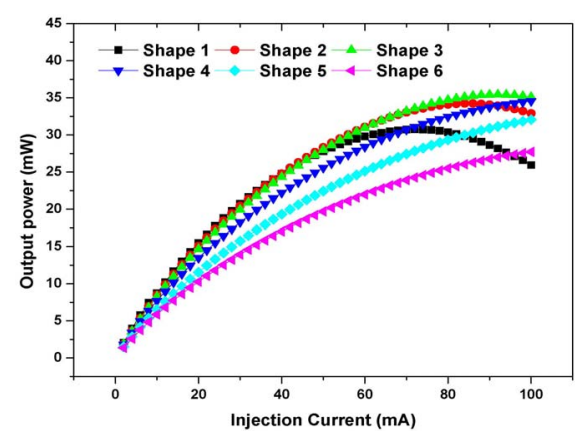

Figure 3. Output powers with injection current for six samples.

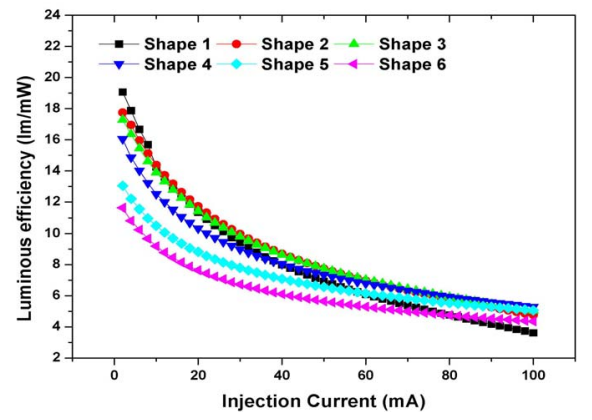

Figure 4. Luminous efficiencies with injection current for six samples.

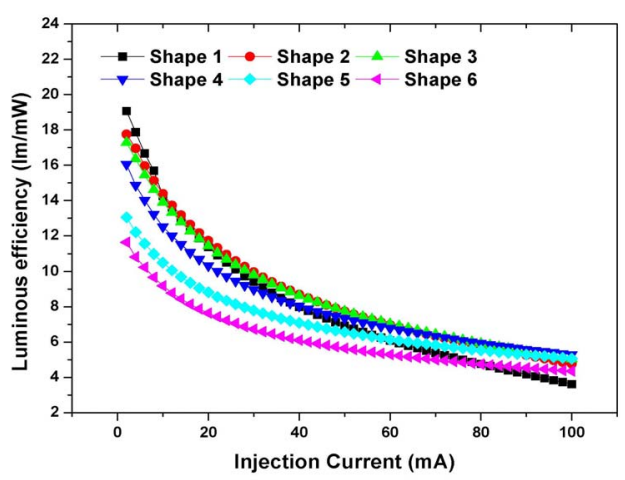

Figure 5. Peak wavelengths with injection current for six samples.

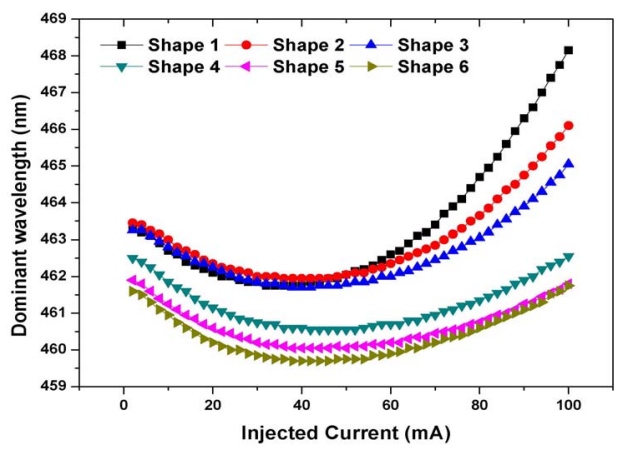

Figure 6. Dominant wavelength with injection current for six samples.

wavelength shifting rate of sample \#1 should be attributed to the current crowding effect, as current is crowded near the P-pad region which could raise the junction temperature and lead to a red shift of emission wavelength [18]. On the other hand, strong built-in electric fields created polarization effects in $\mathrm{InGaN} / \mathrm{GaN}$ multiple quantum well can tilt the band edges, which will produce quantum constrained Stark effects (QCSE) [19], and with the increment of the injection current, the free carriers in multiple quantum well (MQW) increase, the field induced by electrons and holes in the local states will screen the polarized field at a certain extent, and decrease the QCSE, which will overpass the red shift induced by the heat effect, so the peak position shifts to the short wavelength and the blue shift is observed. And band filling of localized states originating from compositional fluctuation of InGaN alloys may attribute to the blue shift [20]. The blue shift values corresponding to samples \#1 \#6 are 1.2, 1, 1.55, 1.25, 1.6 and $1.95 \mathrm{~nm}$, while the red one are $8.05,4.95,4.00,2.2,2.25$ and $2.4 \mathrm{~nm}$, respectively. The red shifts of the former three samples are larger than the latter ones, and the blue shifts are on the contrary. The reason may be that, for the more mesa area, electrons and holes injected in the MQW are larger, which screen the polarized filed strongly, and the band tilts slightly, so the blue shift is larger. At the same time, 
the chip has less crowding effect, and its heat effect reduces, the gap shrinks weakly, so the red shift is smaller. The changes of the dominant wavelength are shown in Figure 6. Similar to the peak wavelength, with increaseing injection current the dominant wavelengths are blue shift firstly, then red, where the blue shifts are $1.55,1.5$, $1.55,1.95,1.85,1.9 \mathrm{~nm}$, and the red are $6.4,4.15,3.35,2$, $1.75,2.05 \mathrm{~nm}$, respectively.

\section{Conclusion}

GaN/InGaN lighting-emitting diodes with six mesa structures are studied. For the more mesa area, V-I characteristics of the $\mathrm{GaN} / \mathrm{InGaN}$ lighting-emitting diodes can be greatly improved by reducing the current crowding. As the chip with more mesa area will countervail the efficient light-emitting area, and the light output power will decrease slightly. One can see that with injection current up to $60 \mathrm{~mA}$ the light output power of GaN-based LED with more mesa area can be improved greatly. Therefore, this mesa pattern will be helpful to the manufacture of the large power LED. This will be considered in our further work. From the changes of the peak and dominant wavelengths, their shift blue firstly, and then red, but the last two chips had a larger blue shift and smaller red shift. Therefore, both considering the optical and electrical properties, the last two mesa structures are the favorable ones for the optoelectronic performance LEDs, where their P-electrode area is larger, and the PN electrodes spacing is shorter.

\section{Acknowledgements}

The authors gratefully acknowledge the financial supports from Yangzhou Science and Technology Development (No: YZ2009092, YZ2010153), and give many thanks to Dr. Yue-Ming Lin and Yangzhou Huaxia Integrated OE System Co., Ltd for their kind help.

\section{REFERENCES}

[1] D. A. Steigerwald, J. C. Bhat, D. Collins, et al., "Illumination with Solid State Lighting Technology," IEEE Journal Selected Topics in Quantum Electronics, Vol. 8, No. 2, 2002, pp. 310-320. doi:10.1109/2944.999186

[2] S. Nakamura, "Growth of $\operatorname{In}_{\mathrm{x}} \mathrm{Ga}_{(1-\mathrm{x})} \mathrm{N}$ Compound Semiconductors and High-Power InGaN/AlGaN Double Heterostructure Violet-Light-Emitting Diodes," Microelectronics Journal, Vol. 25, No. 8, 1994, pp. 651-659. doi:10.1016/0026-2692(94)90131-7

[3] J. J. Wierer, D. A. Steigerwald, M. R. Krames, et al., "High-Power AlGaInN Flip-Chip Light-Emitting Diodes," Applied Physics Letters, Vol. 78, No. 22, 2001, pp. 33793382. doi: $10.1063 / 1.1374499$

[4] Y. J. Lee, P. C. Lin, T. C. Lu, et al., "Dichromatic InGaN-Based White Light Emitting Diodes by Using Laser Lift-Off and Wafer-Bonding Schemes," Applied Physics
Letters, Vol. 90, No. 16, 2007, Article ID: 161115. doi:10.1063/1.2722672

[5] I. Schnitzer and E. Yablonovitch, "30\% External Quantum Efficiency from Surface Textured, Thin-Film LightEmitting Diodes," Applied Physics Letters, Vol. 63, No. 16, 1993, pp. 2174-2176. doi:10.1063/1.110575

[6] X. Guo and E. F. Schubert, "Current Crowding in GaN/ InGaN Light Emitting Diodes on Insulating Substrates," Applied Physics Letters, Vol. 90, No. 8, 2001, p. 4191. doi:10.1063/1.1403665

[7] H. Kim, J. Cho, J. W. Lee, S. Yoon, H. Kim, C. Sone, Y. Park and T.-Y. Seong, "Consideration of the Actual Current-Spreading Length of GaN-Based Light-Emitting Diodes for High-Efficiency Design," IEEE Journal of Quantum Electronics, Vol. 43, No. 8, 2007, pp. 625-632. doi:10.1109/JQE.2007.900262

[8] S.-R. Jeon, Y.-H. Song, H.-J. Jang and G. M. Yang, "Lateral Current Spreading in GaN-Based Light-Emitting Diodes Utilizing Tunnel Contact Junctions," Applied Physics Letters, Vol. 78, No. 21, 2001, pp. 3265-3267. doi:10.1063/1.1374483

[9] H. Kim, J. Cho, J. W. Lee, S. Yoon, H. Kim, C. Sone and Y. Park, "Enhanced Light Extraction of GaN-Based LightEmitting Diodes by Using Textured N-Type GaN Layers," Applied Physics Letters, Vol. 90, No. 16, 2007, Article ID: 161110.

[10] X. Guo and E. F. Schubet, "Current Crowding in GaN/ InGaN Light Emitting Diodes," Applied Physics Letters, Vol. 90, No. 8, 2001, pp. 4191-4195. doi:10.1063/1.1403665

[11] X. Guo, Y.-L. Li and E. F. Schubert, "Current Crowding and Optical Saturation Effects in GaInN/GaN Light-Emitting Diodes Grown on Insulating Substrates," Applied Physics Letters, Vol. 78, No. 21, 2001, pp. 3337-3339. doi:10.1063/1.1372359

[12] V. K. Malyutenko, O. Yu. Malyutenko, A. D. Podoltsev, and I. N. Kucheryavaya, "Current Crowding in InAsSb Light-Emitting Diodes," Applied Physics Letters, Vol. 79, No. 25, 2001, pp. 4228-4230. doi:10.1063/1.1424065

[13] K. A. Bulashevich, I. Yu. Evstratov, V. F. Mymrin and S. Yu. Karpov, "Current Spreading and Thermal Effects in Blue LED Dice," Physica Status Solidi C, Vol. 4, No. 1, 2007, pp. 45-48. doi:10.1002/pssc.200673502

[14] X. J. Zhang, R. X. Yang and J. H. Wang, "Enhanced Light Outpower in InGaN/GaN Light-Emitting Diodes with a High Reflective Current Blocking Layer," Journal of Semiconductors, Vol. 33, No. 7, 2012, Article ID: 074 008. doi:10.1088/1674-4926/33/7/074008

[15] Y. J. Dong, J. B. Zhang, H. T. Chen and X. H. Zeng, "Performance of Power Omnidirectimal Reflector LED," Acta Physica Sinica, Vol. 60, No. 7, 2011, Article ID: 077803.

[16] L. Lei, X. H. Zeng, Y. P. Fan and Y. Zhang, "Optimization of Multiple Quantum Well Structure for GaN-Based Blue Light Emitting Diode," Journal of Optoelectronics Laser, Vol. 22, No. 9, 2011, pp. 1326-1331.

[17] L. M. Chang, S. J. Chang, Z. Y. Jiao, et al., "Enhanced Current Spreading for GaN-Based Side-View LEDs by Adding an Metallic Stripe across the Long Side of the 
Chip," IEEE Photonics Technology Letters, Vol. 24, No. 3, 2012, pp. 1412-1414. doi:10.1109/LPT.2012.2205236

[18] S.-H. Han, D.-Y. Lee, S.-J. Lee, et al., "Effect of Electron Blocking Layer on Efficiency Droop in InGaN/GaN Multiple Quantum Well Light-Emitting Diodes," Applied Physics Letters, Vol. 94, No. 23, 2009, Article ID: 231123. doi:10.1063/1.3153508

[19] Y. D. Qi, H. Liang, D. Wang, et al., "Comparison of Blue and Green InGaN/GaN Multiple Quantum Well Light-
Emitting Diodes Grown by Metalorganic Vapor Phase Epitaxy," Applied Physics Letters, Vol. 86, No. 10, 2005, Article ID: 101903. doi:10.1063/1.1866634

[20] Y. Yang, X. A. Cao and C. H. Yan, "Rapid Efficiency Roll-Off in High-Quality Green Light-Emitting Diodes on Freestanding GaN Substrates," Applied Physics Letters, Vol. 94, No. 4, 2009, Article ID: 041117. doi:10.1063/1.3077017 